\title{
MBE growth and characterization of a II-VI distributed Bragg reflector and microcavity lattice-matched to $\mathrm{MgTe}$
}

\author{
J.-G. Rousset, J. Kobak, T. Słupinski, T. Jakubczyk, P. Stawicki, E. Janik, \\ M. Tokarczyk, G. Kowalski, M. Nawrocki, W. Pacuski \\ Institute of Experimental Physics, Faculty of Physics, University of Warsaw, ul. Hoża 69, \\ PL-00-681 Warszawa, Poland
}

\begin{abstract}
We present the realization and characterization of a 20 fold, fully latticematched epitaxial distributed Bragg reflector based on $(\mathrm{Cd}, \mathrm{Zn}) \mathrm{Te}$ and $(\mathrm{Cd}, \mathrm{Zn}, \mathrm{Mg}) \mathrm{Te}$ layers. We also present a microcavity based on $(\mathrm{Cd}, \mathrm{Zn}, \mathrm{Mg}) \mathrm{Te}$ containing a $(\mathrm{Cd}, \mathrm{Zn}) \mathrm{Te}$ quantum well. Reflectivity spectra, photoluminescence in real space and in far field are presented.

Keywords: Molecular beam epitaxy, Cadmium compounds, Zinc compounds, Tellurides, Semiconducting II-VI materials, Magneto-optic materials PACS: 78.20.Ci, 78.67.Pt, 68.37.Hk, 78.55.Et
\end{abstract}

\section{Introduction}

In III-V semiconductor compounds, the lattice-matching between GaAs and AlAs allows for the growth of monolithic structures and to realize devices for optoelectronics [1, 2]. For II-VI compounds, the challenge in designing and realizing epitaxial distributed Bragg reflectors (DBRs) and microcavities relies on the compromise between opposite constrains: the lattice-matching and a high refractive index contrast. Lattice-matched DBRs based on II-VI compounds have been reported so far for four substrates (or buffers): GaAs [3], InP [4, ZnTe [5], and $(\mathrm{Cd}, \mathrm{Zn}) \mathrm{Te}$ [6, 7]. For this last one, the substrate was $\mathrm{Cd}_{0.88} \mathrm{Zn}_{0.12} \mathrm{Te}$ and the DBR was based on $(\mathrm{Cd}, \mathrm{Mg}) \mathrm{Te}$ and $(\mathrm{Cd}, \mathrm{Mn}) \mathrm{Te}$ layers grown on the $(\mathrm{Cd}, \mathrm{Zn}) \mathrm{Te}$ buffer. In such a structure, the presence of Mn, which has strong magnetic properties, in the DBRs layers can be a disturbance if one wants to explore magnetooptical effects related to quantum wells [8, 10, 9]. We present here the realization of unstrained DBR lattice-matched to $\mathrm{MgTe}$, based on the stack of $(\mathrm{Cd}, \mathrm{Zn}, \mathrm{Mg}) \mathrm{Te}$ layers with various $\mathrm{Mg}$ contents. Enhancements in the design of the DBR led us to the realization and characterization of a monolithic, optically active microcavity containing a lattice-matched $\mathrm{Cd}_{0.86} \mathrm{Zn}_{0.14} \mathrm{Te}$ quantum well (QW) surrounded by good quality DBRs.

As shown in Fig. 1 , our choice of a structure lattice-matched to MgTe is justified by the possibility of tuning the refractive index and the band gap of 


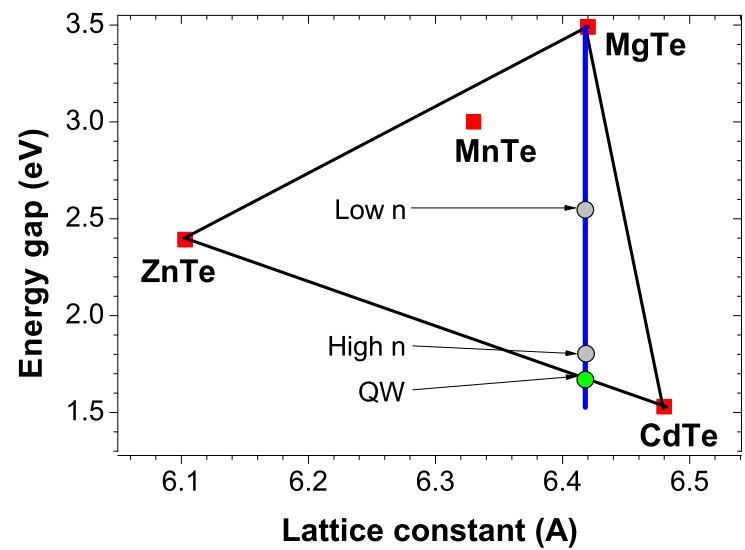

Figure 1: The choice of having a structure lattice-matched to MgTe allows to tune the refractive index contrast through $\mathrm{Mg}$ content of the layers without changing the lattice constant. Line: MgTe lattice constant - dots: high and low refractive index layers, QW.

the layers independently on the lattice parameter, depending only on the $\mathrm{Mg}$ content. With this concept, the whole structure including DBRs and QW can be lattice-matched.

The DBR, microcavity and QW were grown by molecular beam epitaxy (MBE) technique. The MBE machine provided by SVT Associates consists of two growth chambers (for III-V and II-VI semiconductor compounds), each equipped with a reflectivity setup for in situ measurements. Monitoring the reflectivity spectra during the growth allows us to verify the growth rate and thickness of the layers, which is a crucial parameter for the growth of microcavities or DBRs [11, 12, 13, 14].

The structures are grown on a GaAs:Si substrate (100) at the temperature 
of $346^{\circ}$. $\quad \mathrm{A} \mathrm{Cd}_{0.86} \mathrm{Zn}_{0.14} \mathrm{Te}$ buffer about $800 \mathrm{~nm}$ thick is grown to relax the strain due to the lattice mismatch with GaAs. The lattice parameter and the composition of the buffer was estimated in-situ from streaks spacing on the RHEED (reflection high energy electron diffraction) pattern.

\section{Distributed Bragg Reflector}

The DBR containing 20 pairs of low and high refractive index layers was designed so that the center of the stopband is at a wavelength of $\lambda_{0} \approx 900 \mathrm{~nm}$. The layers of the DBR are made of $\lambda / 4 \mathrm{n}$ layers of $\mathrm{Cd}_{0.86} \mathrm{Zn}_{0.14}$ Te for the high refractive index and a 20 fold $\mathrm{Cd}_{0.86} \mathrm{Zn}_{0.14} \mathrm{Te} / \mathrm{MgTe}$ superlattice for the low refractive index. The growth times of the superlattice layers are set to have an effective concentration of $50 \% \mathrm{Mg}$ in the resulting digital alloy. The structure grown was observed by scanning electron microscope (SEM) imaging. As presented in Fig. 2 the layers thicknesses are regular and the interfaces are smooth.

The results from reflectivity measurements compared to the simulation by the transfer matrix method [15] are presented in Fig. 3 The DBR exhibit a maximum reflectivity above $95 \%$. The stopband width measured is $\approx 70 \mathrm{~nm}$ which allows us to evaluate the refractive index contrast using the formula [15] given in eq. 11, where $\Delta \lambda$ sets for the stopband width, $\lambda_{0}$ the center of the stopband, $\tilde{n}$ the average refractive index.

$$
\frac{\Delta \lambda}{\lambda_{0}}=\frac{4}{\pi} \sin ^{-1}\left(\frac{\left|n_{2}-n_{1}\right|}{n_{1}+n_{2}}\right) \approx \frac{2}{\pi} \frac{\Delta n}{\tilde{n}}
$$

Considering that the average refractive index [16] is $\tilde{n} \approx 2.8$, the refractive index contrast is $\Delta n \approx 0.34$.

$\mathrm{X}$-ray diffraction measurements and simulation show that the whole structure (except the substrate) is matched to the lattice constant of MgTe [17] (lattice mismatch $\Delta a / a<0.3 \%$ ) (see Fig. 4 ).

\section{Quantum wells}

In order to obtain a fully lattice-matched microcavity, we designed a $\mathrm{Cd}_{0.86} \mathrm{Zn}_{0.14} \mathrm{Te}$ QW with $\mathrm{Cd}_{0.77} \mathrm{Zn}_{0.13} \mathrm{Mg}_{0.1} \mathrm{Te}$ barriers. Fig. 5 shows photoluminescence spectrum of this ternary QW measured at liquid helium temperature and compared to the photoluminescence spectrum of a CdTe QW.

The PL amplitude of the ternary $\mathrm{Cd}_{0.86} \mathrm{Zn}_{0.14} \mathrm{Te} \mathrm{QW}$ is twice smaller than for the strained CdTe QW, however the half peak width is about $10 \mathrm{meV}$ $(4 \mathrm{~nm})$ for both QWs.

\section{Microcavity with quantum well}

In order to obtain a fully lattice-matched microcavity embedding an optically active QW, the design of the DBR previously presented was slightly modified. The thicknesses of the layers are designed so that the center of the stopband 


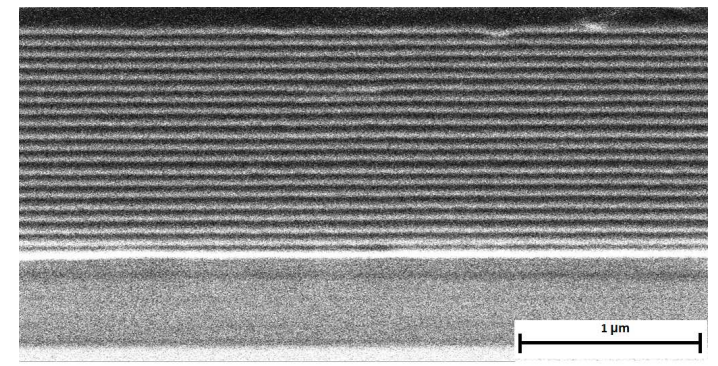

Figure 2: SEM imaging of the DBR. 


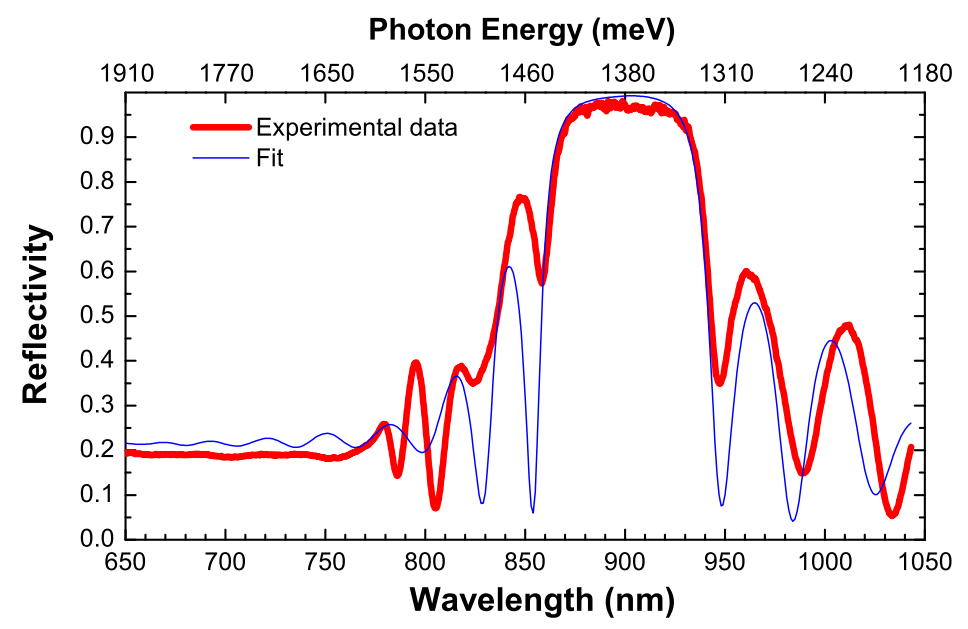

Figure 3: Reflectivity spectra: measurement and simulation at room temperature. The maximum reflectivity is above $95 \%$, the stopband is $\approx 70 \mathrm{~nm}$ wide giving a refractive index contrast $\Delta n \approx 0.34$.

is at $\lambda_{0}=720 \mathrm{~nm}$ corresponding to the emission of the $\mathrm{Cd}_{0.86} \mathrm{Zn}_{0.14} \mathrm{Te} \mathrm{QW}$. In addition, the insertion of the optically active $\mathrm{Cd}_{0.86} \mathrm{Zn}_{0.14} \mathrm{Te} \mathrm{QW}$ requires increasing the energy gap of the high refractive index layer so that it also plays 


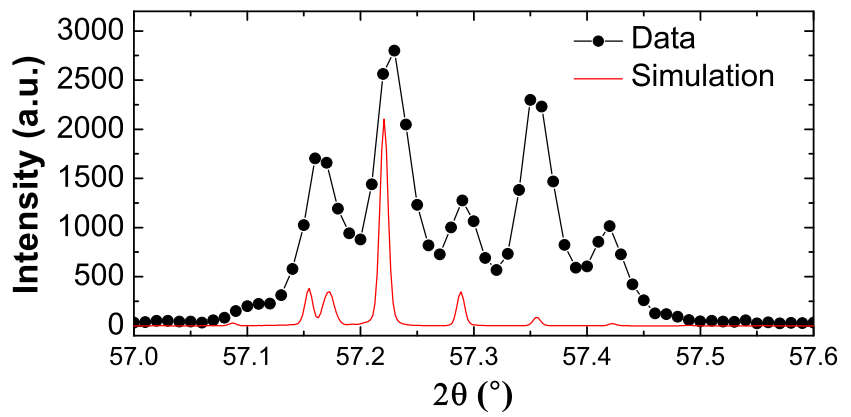

Figure 4: X-ray diffraction measurement (dots) and simulation (solid line) assuming that the structure is relaxed and lattice-matched to $\mathrm{MgTe}$.

the role of the barrier for the QW and does not absorb the QWs emission. This is achieved by adding a small amount of $\mathrm{Mg}$ in the high refractive index layer: $\mathrm{Cd}_{0.77} \mathrm{Zn}_{0.13} \mathrm{Mg}_{0.1} \mathrm{Te}$. In order to keep a relatively high refractive index contrast, the effective $\mathrm{Mg}$ content in the low refractive index layer (superlattice) has also been increased close to $60 \%$. As presented in Fig. 6, the whole structure consists of a $20 \mathrm{~nm}$ thick QW at the center of a $\lambda / n$ microcavity embedded between two 17 fold DBRs.

Reflectivity and photoluminescence spectra have been measured at $T=8 \mathrm{~K}$. 


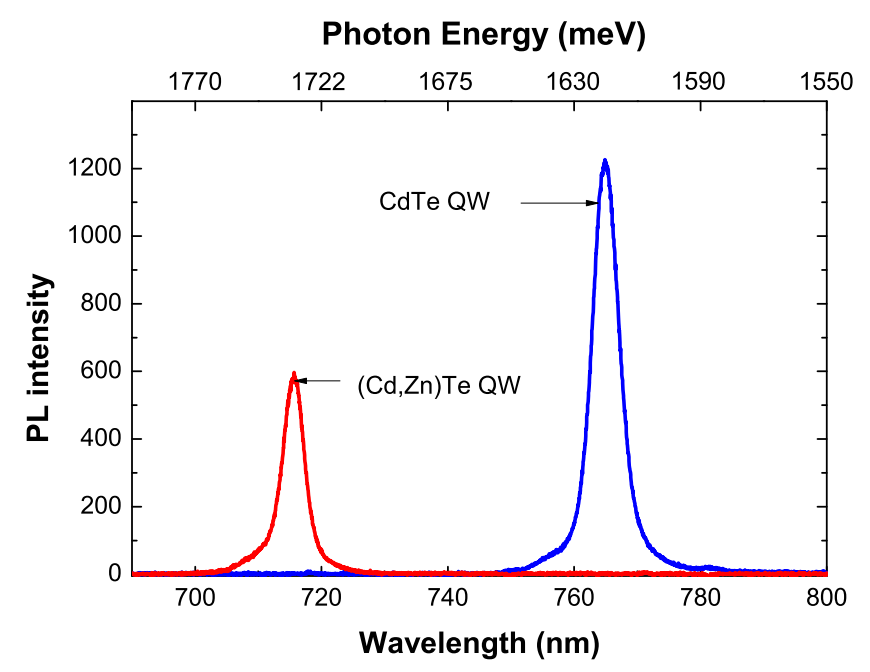

Figure 5: Photoluminescence of CdTe QW (strained) and unstrained $\mathrm{Cd}_{0.86} \mathrm{Zn}_{0.14} \mathrm{Te} \mathrm{QW}$. In both cases the QW is $10 \mathrm{~nm}$ thick, the barrier is $\mathrm{Cd}_{0.77} \mathrm{Zn}_{0.13} \mathrm{Mg}_{0.1} \mathrm{Te}$ and the width (FWHM) of PL peak is about $10 \mathrm{meV}(4 \mathrm{~nm})$.

As shown in Fig. 7, the microcavity exhibit a maximum reflectivity of about $90 \%$. The quality factor is $Q=\frac{\lambda}{\Delta \lambda} \approx 150$.

The photoluminescence measurements presented in Fig. 8 show the tuning of the cavity mode to the emission of the QW resulting in the enhancement of the emission intensity at $\lambda_{0}=716 \mathrm{~nm}$, in agreement with our expectation. Different values of the detuning are obtained for different places on the sample. Indeed, the sample was not rotated during growth which results in a gradient 
of the layers' thicknesses.

Far field PL measurements were also conducted (Fig. 91) and show the dependence on the angle of the emission from the cavity whereas the emission from the QW remains unchanged. It shows that in the a microcavity, exciton dispersion is much weaker than photon dispersion. This measurement was realized using the Fourier plane imaging method [18, 7]. By using this technique, adding more quantum wells in the cavity, having QW lines more sharp, increasing quality factor of the microcavity, and using high excitation power we expect to observe Bose-Einstein condensation of exciton polaritons [7]. In present study we are very far from this conditions.

\section{Conclusion}

The growth of DBR, QW and microcavity lattice-matched to MgTe based on a $\mathrm{Cd}_{0.86} \mathrm{Zn}_{0.14}$ Te buffer was presented. This allowed the tuning of the refractive index and energy gap through the $\mathrm{Mg}$ content of the layers. The 20 fold DBR exhibits a reflectivity above $95 \%$. X-ray diffraction measurements show that the whole structure is lattice-matched to MgTe. The tuning of the emission from the $\mathrm{Cd}_{0.86} \mathrm{Zn}_{0.14} \mathrm{Te} \mathrm{QW}$ to the cavity mode results in an enhancement of the photoluminescence at $\lambda_{0}=716 \mathrm{~nm}$. The far field emission shows the dependence of the emission from the cavity on the angle of the propagating light. The realization of such a microcavity is a first step toward the realization of a vertical cavity surface emitting laser (VCSEL).

\section{Acknowledgement}

This work was supported by Polish public funds in years 2011 - 2014 (NCBiR project LIDER). Research was carried out with the use of CePT, CeZaMat and NLTK infrastructures financed by the European Union - the European Regional Development Fund within the Operational Programme "Innovative economy" for 2007-2013.

\section{References}

[1] J. M. Gérard, B. Sermage, B. Gayral, B. Legrand, E. Costard and V. Thierry-Mieg, Physical Review Letters 81, 1110 (1998).

[2] A. Dousse, J. Suffczynski, A. Beveratos, O. Krebs, A. Lemaitre, I. Sagnes, J. Bloch, P. Voisin and P. Senellart, Nature 466 (2010) 217.

[3] C. Kruse, S.M. Ulrich, G. Alexe, E. Roventa, R. Kröger, B. Brendemühl, P. Michler, J. Gutowski, D. Hommel, Physica Status Solidi (b) 241, (2004) 731.

[4] S.P. Guo, O. Maksimov, M.C. Tamargo, F.C. Peiris, J.K. Furdyna, Applied Physics Letters 77 (2000) 4107. 
[5] W. Pacuski, C. Kruse, S. Figge, D. Hommel, Applied Physics Letters 94 (2009) 191108.

[6] L.S. Dang, D Heger, R. André, F. Boeuf, R. Romestain, Physical Review Letters 81 (1998) 3920.

[7] J. Kasprzak, M. Richard, S. Kundermann, A. Baas, P. Jeambrun, J. M. J. Keeling, F. M. Marchetti, M. H. Szymańska, R. André, J. L. Staehli, V. Savona, P. B. Littlewood, B. Deveaud and Le Si Dang, Nature 443 (2006) 409.

[8] H. Ulmer-Tuffigo, F. Kany, G. Feuillet, R. Langer, J. Bleuse and J. L. Pautrat, Journal of Crystal Growth 159 (1996) 605.

[9] A. Brunetti, M. Vladimirova, D. Scalbert, R. André, D. Solnyshkov, G. Malpuech, I. Shelykh and A. V. Kavokin. Physical Review B 73 (2006) 205337.

[10] A. Brunetti, M. Vladimirova., D. Scalbert and R. Andre, Physica Status Solidi (c) 2 (2005) 3876.

[11] C. Kruse, G. Alexe, M. Klude, H. Heinke, and D. Hommel, Physica Status Solidi (b) 229 (2002) 111.

[12] H. P. D. Schenk, P. de Mierry, P. Vennéguès, O. Tottereau and M. Laügt, M. Vaille, E. Feltin, B. Beaumont, P. Gibart, S. Fernández and F. Calle. Applied Physics Letters 80 (2002) 174.

[13] M. Mizutani, F. Teramae, O. Kobayashi, S. Naritsuka and T. Maruyama, Physica Status Solidi (c) 3 (2006) 659.

[14] K. Biermann and E.A. Cerda-Méndez and M. Höricke and P.V. Santos and R. Hey, Journal of Crystal Growth 323 (2011) 56.

[15] P. Yeh, Optical Waves in Layered Media, Wiley, New York (1988).

[16] D. T. F. Marple, Journal of Applied Physics 35 (1964) 539.

[17] E. Dynowska and E. Janik and J. Bąk-Misiuk and J. Domagała and T. Wojtowicz and J. Kossut, Journal of Alloys and Compounds 286 (1999) 276.

[18] T. Jakubczyk, W. Pacuski, P. Duch, P. Godlewski, A. Golnik, C. Kruse, D. Hommel, J. A. Gaj, Central European Journal of Physics 9 (2011) 428. 


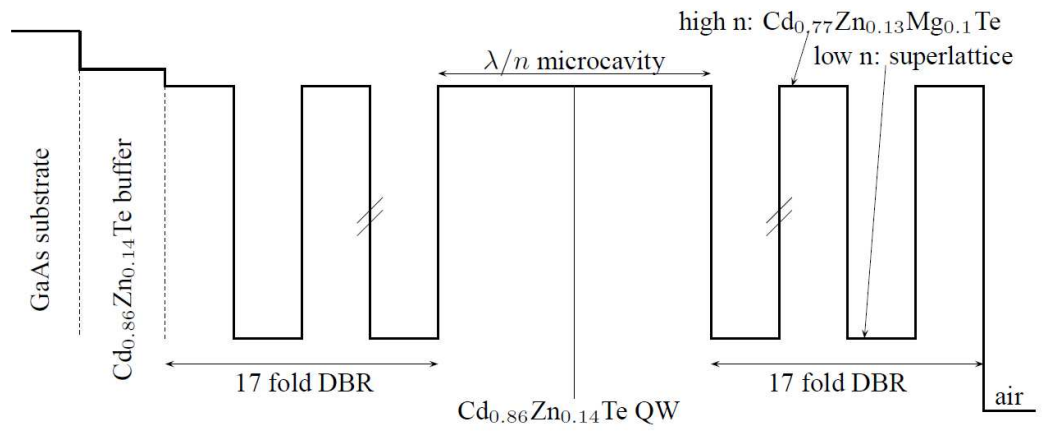

Figure 6: Structure of the microcavity. Vertical axis: refractive index, horizontal axis: depth of the sample. 


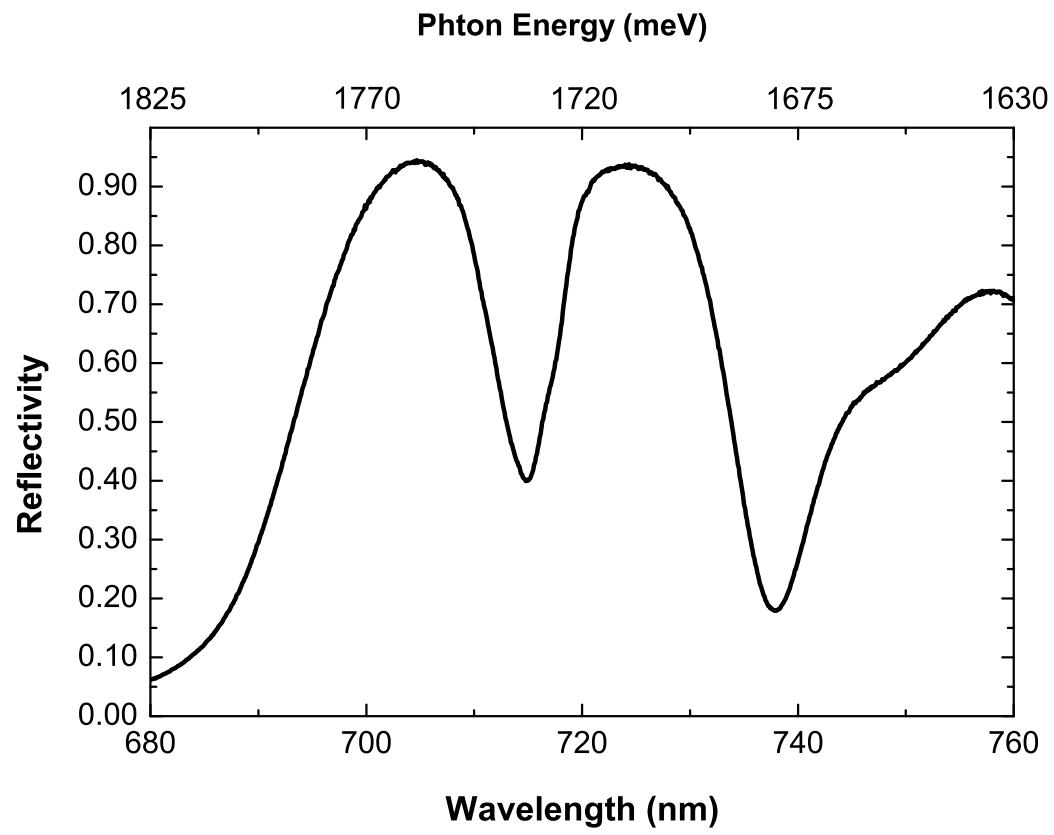

Figure 7: Reflectivity spectrum of the microcavity measured at $T=8 \mathrm{~K}$. The quality factor of the cavity is $Q \approx 150$ 


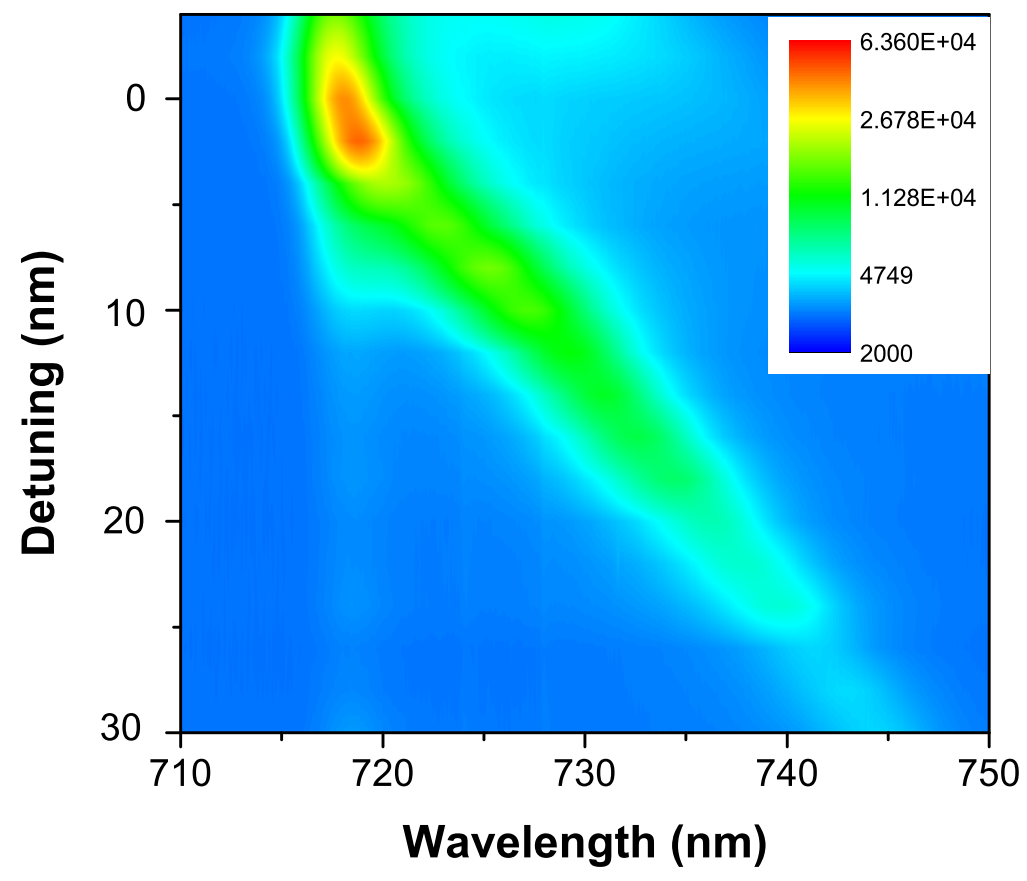

Figure 8: Map showing the photoluminescence as a function of the wavelength for various values of the detuning. 


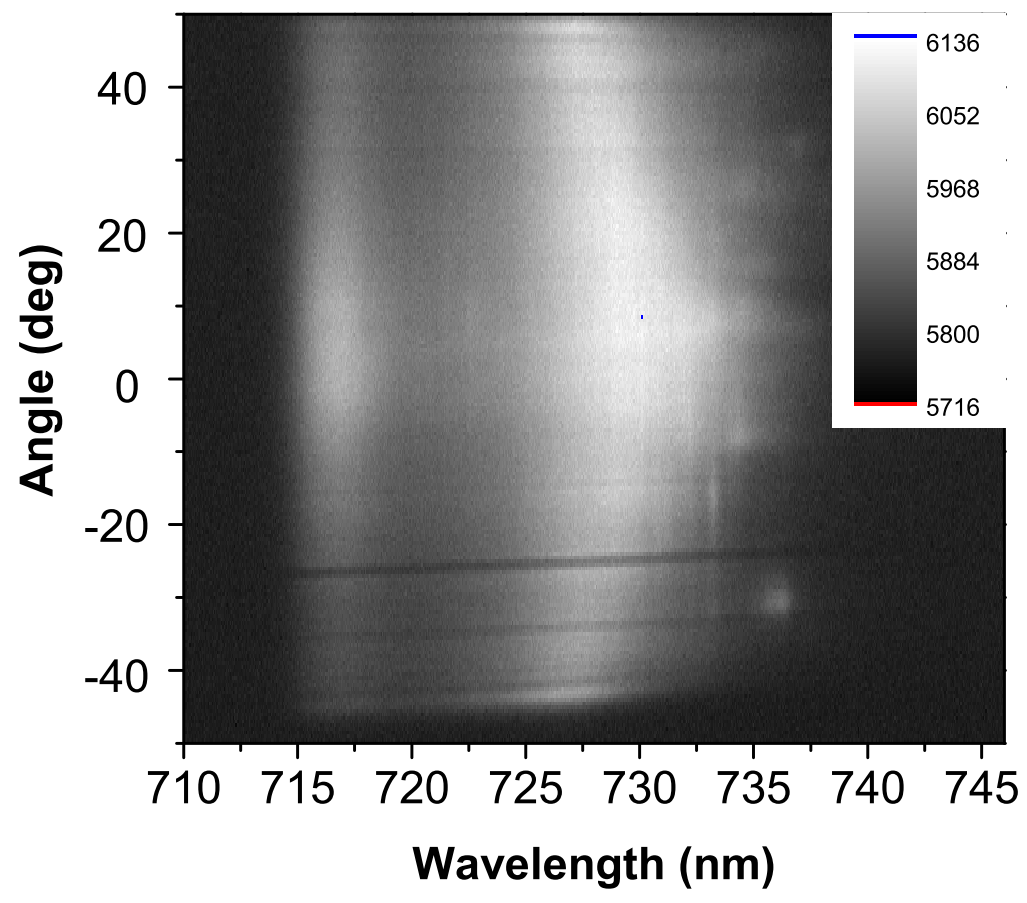

Figure 9: Far field emission. The wavelength of the cavity mode depends on the propagation direction of the light. The emission from the QW remains at the same wavelength. 